\title{
GENERALIZED MEAN VALUE THEOREMS OF THE DIFFERENTIAL CALCULUS
}

\author{
J. B. DIAZ and R. VÝBORNÝ* \\ (Received 3 March 1973; revised 12 April 1974) \\ Communicated by E. Strezelecki
}

\begin{abstract}
Two generalized mean value theorems, for functions with values in a linear locally convex topological space, are proved, as consequences of two theorems for real valued functions of a real variable.
\end{abstract}

\section{Introduction}

The classical mean value theorem of the differential calculus states that for a real valued function $f$, defined and continuous on a finite closed interval $[a, b]$, where $a<b$, and which has a derivative on the open interval $(a, b)$, there is a mean value $c$, such that $a<c<b$, and

$$
f(b)-f(a)=f^{\prime}(c)(b-a) .
$$

In the mathematical literature there appear several valid generalizations of this theorem to vector valued functions of a real variable; these generalizations are, roughly speaking, of two sorts:

(a) the equation ( 0 ) is replaced by an inequality involving norms if the vector valued function is denoted by $x(t)$, then the inequality reads $\|x(b)-x(a)\| \leqq$ $\left.\left\|x^{\prime}(c)\right\|(b-a)\right\}$,

(b) the equation (0) is replaced by the statement that $\frac{x(b)-x(a)}{b-a}$ lies in the closed convex hull of the range of the derivative.

These two types of generalizations, which are not altogether unrelated,

* This author gratefully acknowledges that this research was carried out when he was visiting Rensselaer Polytechnic Institute and was supported by the Albert Einstein Chair of Science. 
have been used, in the literature, in connection with l'Hôspital's rule, and with uniqueness theorems for differential equations.

Instances of (a) are to be found in Aziz and Diaz (1963), and, of (b), in Wazewski (1953) and pp. 201-202 of Averbuch and Smoljanov (1967).

There is another feature to these generalizations, namely, the occurrence of an exceptional set $E \subset(a, b)$, where the function in question is either not required to be differentiable, or the values of the derivative are not taken into account. The classical mean value theorem of the differential calculus is no longer true even is the exceptional set consists only of one number (consider, for example $|x|$ on $[-1,1])$; however, in the generalizations of type (a) and (b), the exceptional set can be denumerable, and even certain types of uncountable sets are permitted.

The present results were obtained in attempting to find out just how general this exceptional set $E$ could be allowed to be, while still having a valid generalization of the mean value theorem.

The results for vector valued functions, in section 3 , are based upon the preliminary results for real valued finctions (section 2), where the real valued function $f$ is assumed to be differentiable outside the exceptional set. In this respect, the present results are not the most general, since Wazewski (1953) and Mlak (1957) employed Dini derivates; however, Theorem 3 and Theorem 4 include, as special cases, Theorem 2 of Mlak (1957), and the mean value theorems in Averbuch and Smoljanov (1967) and Dieudonné (1960). Other versions of the mean value theorem were given by McLeod (1964-65); generally speaking, he uses two exceptional sets $M, N$. The set $M$ is always a countable set, where the (right or left hand) derivative need not exist; the set $N$ is always a set of measure zero, and the values of $x^{\prime}(t)$ on $N$ are not taken into account. McLeod's results, when the values of the function lie in a finite dimensional space, are of particular interest.

The general approach employed, in the proof of Theorem 1, for a real valued function, uses the completeness of the field of real numbers, in much the same way as it is used in the "creeping lemma" of Moss and Roberts (1968). In the present paper, the vector valued case is reduced to the real valued case (as in Wazewski (1953) and Mlak (1957)) by using the well known fact that a convex closed set, in a real linear locally convex topological space, is the intersection of all closed half-spaces containing the convex set.

\section{Real valued functions}

This section contains two theorems for real valued functions of a real variable.

THEOREM 1. If

(1) $f$ is a real valued function defined on a closed, bounded interval $[a, b]$, with $a<b$; and $f$ is continuous at $a$ and $b$, from the right and from the left, respectively; 
(2) $g$ is a real valued nondecreasing continuous function on $[a, b]$;

(3) $E$ is a subset of $(a, b)$ such that, for every $\varepsilon>0$, there is a sequence of disjoint, open intervals $\left(\alpha_{k}, \beta_{k}\right), k=1,2, \cdots$, with $\alpha_{k}<\beta_{k}$, and $\left[\alpha_{k}, \beta_{k}\right] \subset[a, b]$, such that

$$
E \subset \bigcup_{k=1}^{\infty}\left(\alpha_{k}, \beta_{k}\right), \text { and, also, } \sum_{k=1}^{\infty}\left|f\left(\beta_{k}\right)-f\left(\alpha_{k}\right)\right|<\varepsilon ;
$$

(4) $f$ has $a$, finite or infinite, derivative for $t \in(a, b)-E ; g$ has a finite derivative for $t \in(a, b)-E$, and there is a real number $M$ such that

$$
f^{\prime}(t) \leqq M g^{\prime}(t)
$$

for $t \in(a, b)-E$;

then one has

$$
f(b)-f(a) \leqq M^{+}[g(b)-g(a)],
$$

where $M^{+}=\max (0, M)$.

Proof. Without loss of generality, it can be assumed that $g$ is strictly increasing, and that $g^{\prime}(t)>0$ for $t \in(a, b)-E$, by simply replacing the given $g(x)$ by $g(x)+\eta x$, where $\eta>0$ is any fixed, but arbitrary, positive number. The final conclusion of the theorem, upon completion of the proof, will then read

$$
f(b)-f(a) \leqq\left(M^{+}+\eta\right)(g(b)-g(a)) ;
$$

but, since $\eta>0$ is arbitrary, the desired inequality (1) follows from this. The precise moment in the proof, at which the additional assumptions $g^{\prime}(t)>0$, $g^{\prime}(t)$ finite, for $t \in(a, b)-E$, are needed, will be explicitly indicated below in the course of the argument.

In trying to follow the argument, the reader may do well to keep in mind the intuitive idea of the proof, which may be informally described as "creeping from $a$ to $b$, using only the numbers $x$, with $a \leqq x \leqq b$, which do not belong to the set $\bigcup_{k=1}^{\infty}\left(\alpha_{k}, \beta_{k}\right)$ " (the present situation is to be compared with that in Moss and Roberts (1968), where there is no exceptional set $E$ ).

Let $\varepsilon>0$, and $\left(\alpha_{k}, \beta_{k}\right), k=1,2, \cdots$, be the corresponding sequence of intervals appearing in hypothesis (3). Let $S$ be the set of all numbers $\xi$, with $a \leqq \xi \leqq b$, satisfying the following two conditions: and,

(A) the number $\xi$ is not in $\bigcup_{k=1}^{\infty}\left(\alpha_{k}, \beta_{k}\right)$;

(B) for all $x$, with $a \leqq x \leqq \xi$, and $x$ not in $\bigcup_{k=1}^{\infty}\left(\alpha_{k}, \beta_{k}\right)$, it is true that

$$
f(x)-f(a) \leqq\left(M^{+}+\varepsilon\right)[g(x)-g(a)]+\sum_{\alpha_{k}<x}\left|f\left(\beta_{k}\right)-f\left(\alpha_{k}\right)\right|+\varepsilon .
$$

Clearly, $a$ itself is in $S$; since, in the first place, $a$ is not in $\bigcup_{k=1}^{\infty}\left(\alpha_{k}, \beta_{k}\right)$, and 
inequality (2) holds for $x=a$, with the sum $\Sigma_{z_{k}<a}\left|f\left(\beta_{k}\right)-f\left(\alpha_{k}\right)\right|$, in (2), being, naturally, interpreted as zero.

The whole point of the argument below is to show that the number $b$, which is, obviously, not in the $\bigcup_{k=1}^{\infty}\left(\alpha_{k}, \beta_{k}\right)$, is also in the set $S$. Once this is done, the desired conclusion, (1), follows immediately by using the inequality (2), for $x=b$, and employing the arbitrariness of $\varepsilon>0$.

The following lemma will be used several times.

Lemma. If $\alpha_{j} \in S$, for some positive integer $j$, then, also, $\beta_{j} \in S$.

PRoof. Since $\alpha_{j} \in S$, it is true that for any $x$, with $a \leqq x \leqq \alpha_{j}$, and $x$ not in $\bigcup_{k=1}^{\infty}\left(\alpha_{k}, \beta_{k}\right)$, the inequality (2) is valid. Therefore, since $\beta_{j}$ does not belong to the $\bigcup_{k=1}^{\infty}\left(\alpha_{k}, \beta_{k}\right)$, in order to show that $\beta_{j} \in S$, it only remains to be shown, in view of the definition of the set $S$, that inequality (2) holds with $x$ replaced by $\beta_{j}$.

Obviously, using the inequality (2) for $x=\alpha_{j}$, plus the fact that the function $g$ is nondecreasing on $[a, b]$, it follows that

$$
\begin{aligned}
& f\left(\beta_{j}\right)-f(a) \leqq f\left(\beta_{j}\right)-f\left(\alpha_{j}\right)+f\left(\alpha_{j}\right)-f(a) \\
& \leqq f\left(\beta_{j}\right)-f\left(\alpha_{j}\right)+\left(M^{+}+\varepsilon\right)\left[g\left(\alpha_{j}\right)-g(a)\right]+\sum_{\alpha_{k}<\alpha_{j}}\left|f\left(\beta_{k}\right)-f\left(\alpha_{k}\right)\right|+\varepsilon \\
& \leqq\left|f\left(\beta_{j}\right)-f\left(\alpha_{j}\right)\right|+\left(M^{+}+\varepsilon\right)\left[g\left(\alpha_{j}\right)-g(a)\right]+\sum_{\alpha_{k}<\alpha_{j}}\left|f\left(\beta_{k}\right)-f\left(\alpha_{k}\right)\right|+\varepsilon \\
& \leqq\left(M^{+}+\varepsilon\right)\left(g\left(\beta_{j}\right)-g(a)\right)+\sum_{\alpha_{k}<\beta_{j}}\left|f\left(\beta_{k}\right)-f\left(\alpha_{k}\right)\right|+\varepsilon .
\end{aligned}
$$

Hence, besides $\beta_{j}$ not: belonging to the $\bigcup_{k=1}^{\infty}\left(\alpha_{k}, \beta_{k}\right)$, it is also true that the inequality (2) is satisfied for all $x, a \leqq x \leqq \beta_{j}$, and $x$ not in $\bigcup_{k=1}^{\infty}\left(\alpha_{k}, \beta_{k}\right)$; and it has been proved that $\beta_{j} \in S$. This completes the proof of the lemma.

Let $s=\sup S$. Obviously, $s \leqq b$. It will now be shown that $a<s$. If $a=\alpha_{j}$ for some positive integer $j$, then, by the lemma, also $\beta_{j} \in S$, and hence $a<\beta_{j} \leqq s$. If, on the other hand, $a \neq \alpha_{j}$ for every $j$, then (see hypothesis (1)), by the continuity of the function $f$ at $a$, it must be true that, for some positive number $\delta<b-a$, the inequality

$$
f(x)-f(a)<\varepsilon
$$

holds, for all $x$ with $a \leqq x \leqq a+\delta$, whether $x$ is in $\bigcup_{k=1}^{\infty}\left(\alpha_{k}, \beta_{k}\right)$ or not (and hence, a fortiori, inequality (2) also holds for such an $x$ ). Two cases arise: either (A) some $\alpha_{j} \in(a, a+\delta)$, or (B) no $\alpha_{j} \in(a, a+\delta)$. In case (A), any such $\alpha_{j}$ must belong to $S$, and $a<\alpha_{j} \leqq s$. In case (B), the number $a+\delta / 2$, say, must belong to $S$, and $a<a+\delta / 2 \leqq s$. Thus, $a<s$, in all cases, as desired.

Next, it will be proved that $s$ is not in $\bigcup_{k=1}^{\infty}\left(\alpha_{k}, \beta_{k}\right)$. Suppose, contrary to what one wishes to prove, that $s \in \bigcup_{k=1}^{\infty}\left(\alpha_{k}, \beta_{k}\right)$; then, $\alpha_{i}<s<\beta_{i}$, for some 
positive integer $i$. By the definition of $s$ as the least upper bound of $S$, there exists a number $\xi$ in $S$ such that $\alpha_{i}<\xi \leqq s$. Since $\alpha_{i}$ is not in $\bigcup_{k=1}^{\infty}\left(\alpha_{k}, \beta_{k}\right)$; and (because $\xi$ is in $S$ ), inequality (2) holds for all numbers $x$, with $x$ not in $\bigcup_{k=1}^{\infty}\left(\alpha_{k}, \beta_{k}\right)$, such that $a \leqq x \leqq \xi$, it follows that inequality (2) holds, a fortiori, for all numbers $x$, with $x$ not in $\bigcup_{k=1}^{\infty}\left(\alpha_{k}, \beta_{k}\right)$, such that $a \leqq x \leqq \alpha_{i}$. Hence, $\alpha_{i} \in S$. But, then, by the lemma, $\beta_{i}$ also belongs to $S$, which contradicts the definition of $s$ as the supremum of the set $S$, because $s<\beta_{i}$.

It will now be shown that $s \in S$. Two possibilities arise: either (A) for some positive integer $n$, it is true that $a<s-1 / n$, and no number $x$, with $s-1 / n \leqq$ $x<s$, belongs to the set $S$; or (B) for every positive integer $n$ such that $a<s-1 / n$, there is a number $x_{n}$, with $s-1 / n \leqq x_{n}<s$, such that $x_{n}$ is in $S$. If possibility $(A)$ holds, in which case $s$ is an "isolated" supremum of the set $S$, then the very definition of $s$, as the supremum of the set $S$, yields that $s \in S$. If possibility (B) holds, then inequality 2) holds for all $x$ such that $a \leqq x \leqq x_{n}$, with $x$ not in $\bigcup_{k=1}^{\infty}\left(\alpha_{k}, \beta_{k}\right)$; and from this, and $\lim _{n \rightarrow \infty} x_{n}=s$, plus the continuity of the functions $f$ and $g$ at $s$ (using the fact that, as was already shown, $s$ is not in $\bigcup_{k=1}^{\infty}\left(\alpha_{k}, \beta_{k}\right)$, and thus $\left.s \in(a, b)-E\right)$,it follows that inequality (2) holds for all $x$ such that $a \leqq x \leqq s$, with $x$ not in $\bigcup_{k=1}^{\infty}\left(\alpha_{k}, \beta_{k}\right)$. Since it is already known that $s$ is not in $\bigcup_{k=1}^{\infty}\left(\alpha_{k}, \beta_{k}\right)$, this means that $s \in S$, as was to be shown.

Finally, it will be shown, by contradiction, that, actually, $s=b$. Suppose, contrary to what one wishes to prove, that $s<b$. Since, $s$ is not in $\bigcup_{k=1}^{\infty}\left(\alpha_{k}, \beta_{k}\right), f$ and $g$ have derivatives at $s$, and

$$
f^{\prime}(s) \leqq M g^{\prime}(s)
$$

It is here where the additional assumptions (compare hypothesis (4), and the remark at the very beginning of this proof) that $g^{\prime}>0$, and $g^{\prime}$ finite, are first used. Then, the strict inequality

$$
f^{\prime}(s)<(M+\varepsilon) g^{\prime}(s)
$$

holds; and, from the definition of the derivative, (whether $f^{\prime}(s)$ is finite or not), it follows that there is a positive number, $\delta=\delta(\varepsilon)$, such that, $s+\delta \leqq b$ and for all $x$ with $s<x \leqq s+\delta$, it is true that

and, hence,

$$
\frac{f(x)-f(s)}{x-s}<(M+\varepsilon)\left(\frac{g(x)-g(s)}{x-s}\right)
$$

$$
f(x)-f(s) \leqq(M+\varepsilon)(g(x)-g(s))
$$

whenever $s \leqq x \leqq s+\delta$.

Using (3), and (2) for $x=s$, one obtains 
$f(x)-f(a)=f(x)-f(s)+f(s)-f(a) \leqq(M+\varepsilon)[g(x)-g(s)]+$

$$
\begin{aligned}
& +\left(M^{+}+\varepsilon\right)[g(s)-g(a)]+\sum_{\alpha_{k}<s}\left|f\left(\beta_{k}\right)-f\left(\alpha_{k}\right)\right|+\varepsilon \leqq \\
& \leqq\left(M^{+}+\varepsilon\right)[g(x)-g(a)]+\sum_{\alpha_{k}<x}\left|f\left(\beta_{k}\right)-f\left(\alpha_{k}\right)\right|+\varepsilon,
\end{aligned}
$$

for all $x$ such that $s \leqq x \leqq s+\delta$, regardless of whether $x$ is, or is not, in $\bigcup_{k=1}^{\infty}\left(\alpha_{k}, \beta_{k}\right)$. Further, since $s \in S$, it follows that $s$ is not in $\bigcup_{k=1}^{\infty}\left(\alpha_{k}, \beta_{k}\right)$, and that (2) holds for all $a \leqq x \leqq s$, with $x$ not in $\bigcup_{k=1}^{\infty}\left(\alpha_{k}, \beta_{k}\right)$. Consequently, in view of this, and of the definition of $s$ as the supremum of $S$, it follows that every number $x$ in the open interval $(s, s+\delta)$ must belong to $\bigcup_{k=1}^{\infty}\left(\alpha_{k}, \beta_{k}\right)$; and, since, the intervals $\left(\alpha_{k}, \beta_{k}\right)$ are disjoint, there must be an integer $j$ such that $(s, s+\delta) \subset\left(\alpha_{j}, \beta_{j}\right)$. Therefore, $\alpha_{j} \leqq s<\beta_{j}$. But, whether $\alpha_{j}<s$ or $\alpha_{j}=s$, it then follows, using the fact that $\alpha_{j}$ is not in $\bigcup_{k=1}^{\infty}\left(\alpha_{k}, \beta_{k}\right)$, plus the inequality (4) above, that $\alpha_{j} \in S$. Then, from the lemma, $\beta_{j}$ also belongs to $S$; which, since $s<\beta_{j}$, contradicts the definition of $s$ as the supremum of $S$.

Since $s=b, s \in S$, the inequality (2) holds for $x=b$, and therefore, using hypothesis (3):

$$
f(b)-f(a) \leqq\left(M^{+}+\varepsilon\right)[g(b)-g(a)]+2 \varepsilon .
$$

Using the arbitrariness of $\varepsilon$, inequality (1) follows. The proof of Theorem 1 is complete.

If hypothesis (3) of Theorem 1 is replaced by the following stronger hypothesis: $\left(3^{\prime}\right) E$ is a subset of $(a, b)$ such that, for every $\varepsilon>0$, there is a sequence of disjoint, open intervals $\left(\alpha_{k}, \beta_{k}\right), k=1,2, \cdots$, with $\alpha_{k}<\beta_{k}$, and $\left[\alpha_{k}, \beta_{k}\right] \subset[a, b]$, such that $E \subset \bigcup_{k=1}^{\infty}\left(\alpha_{k}, \beta_{k}\right)$, and also such that both $\sum_{k=1}^{\infty}\left|f\left(\beta_{k}\right)-f\left(\alpha_{k}\right)\right|<\varepsilon$ and $\sum_{k=1}^{\infty} g\left(\beta_{k}\right)-g\left(\alpha_{k}\right)<\varepsilon$; then the conclusion of Theorem 1 can be improved. Namely, the following theorem holds:

THEOREM 2. If hypotheses (1), (2), (3') and (4) hold, then

$$
f(b)-f(a) \leqq M[g(b)-g(a)] .
$$

Proof. If $M \geqq 0$, then (5) is identical with (1). If $M<0$, consider the auxiliary function $F$, defined by $F(x)=f(x)-M g(x)$. Then, Theorem 1, with the constant $M$ of that theorem taken to be zero, applied to the functions $F$ and $g$, gives that

$$
f(b)-f(a)-M[g(b)-g(a)] \leqq 0,
$$

and the proof is complete.

REMARK 0. For convenience of exposition and writing, it has been explicitly supposed, in hypothesis (3) of Theorem 1, that there is, for each $\varepsilon>0$, an infinite sequence of open intervals $\left(\alpha_{k}, \beta_{k}\right)$, but, it is clear, from a glance at the 
proof of the theorem, that, by slight modifications of the argument, this infinite sequence of open intervals can be allowed to be finite, or, if need be, empty. (A similar remark applies to Theorem 2, and to the later Theorems 3 and 4, but it will not be insisted upon.)

Consider the very special case in which this sequence of intervals is empty, the exceptional set $E$ is also empty, and, in hypothesis (4), both $f$ and $g$ have finite derivatives on $(a, b)$. Then, compare Moss and Roberts (1968), the conclusion, (1), of Theorem 1 can be strengthened, so as to coincide with the conclusion, (5), of Theorem 2 (that is, the number $M^{+}$in (1) can be replaced by just $M)$. The following simple example shows that this "replacement of $M^{+}$by $M^{\prime}$ " cannot be performed, in general, even when there is just a single "exceptional" interval $\left(\alpha_{1}, \beta_{1}\right)$ for every $\varepsilon>0$. Choose $a=0, b=2$, and let $f(x)=1-x$ for $0 \leqq x \leqq 1$, while $f(x)$ is defined arbitrarily for $1<x<2$, but it is required that $f(2)=0$. Further, let $g(x)=x$ for $0 \leqq x \leqq 2$, and $M=-1$. Then, Theorem 1 is applicable, with but a single interval, $\left(\alpha_{1}, \beta_{1}\right)=(1,2)$, for each $\varepsilon>0$. However, inequality (1), in the conclusion of Theorem 1 , is not true when $M^{+}$is replaced by $M$.

REMARK 1. If $f$ and $g$ are continuous throughout the interval $[a, b]$, and the exceptional set $E$ is at most countable, then hypothesis $\left(3^{\prime}\right)$ is satisfied, with a possibly finite or empty system of intervals $\left(\alpha_{k}, \beta_{k}\right)$; compare Remark 0 . The proof is as follows.

If $E$ is empty, then the system of intervals may also be taken to be empty (this is the "empty case" of the proof). Therefore, it will be supposed that $E$ is not empty.

Let

$$
t_{1}, t_{2}, t_{3}, \cdots
$$

be a sequence (finite, or infinite) consisting of all the points of $E$. The sequence of intervals $\left(\alpha_{k}, \beta_{k}\right)$ appearing in hypothesis $\left(3^{\prime}\right)$ of Theorem 2 can be constructed as follows. Since both $f$ and $g$ are continuous at $t_{1}$, there exists an interval $\left(\alpha_{1}, \beta_{1}\right)$, with $a \leqq \alpha_{1}<t_{1}<\beta_{1} \leqq b$, such that both

$$
\begin{gathered}
\left|f\left(\beta_{1}\right)-f\left(\alpha_{1}\right)\right|<\frac{\varepsilon}{2}, \\
g\left(\beta_{1}\right)-g\left(\alpha_{1}\right)<\frac{\varepsilon}{2}
\end{gathered}
$$

and both $\alpha_{1}, \beta_{1}$ are not equal to any $t_{k}, k=1,2, \cdots$. Using mathematical induction, suppose that, for some positive integer $n \geqq 1$, the disjoint open intervals $\left(\alpha_{k}, \beta_{k}\right)$, for $k=1,2, \cdots, n$, have been chosen so that $a \leqq \alpha_{k}<\beta_{k} \leqq b$ and

$$
\sum_{k=1}^{n}\left|f\left(\beta_{k}\right)-f\left(\alpha_{k}\right)\right|<\sum_{k=1}^{n} \frac{\varepsilon}{2^{k}}
$$




$$
\sum_{k=1}^{n} g\left(\beta_{k}\right)-g\left(\alpha_{k}\right)<\sum_{k=1}^{n} \frac{\varepsilon}{2^{k}}
$$

and that $t_{i} \in \bigcup_{k=1}^{n}\left(\alpha_{k}, \beta_{k}\right)$, at least for $i=1, \cdots, n$; and that both $\alpha_{k}, \beta_{k}$ are not equal to any $t_{k} ; k=1,2, \cdots$. There are two possibilities: either $E$ is, or is not, contained in $\bigcup_{k=1}^{n}\left(\alpha_{k}, \beta_{k}\right)$. If $E$ is contained in $\bigcup_{k=1}^{n}\left(\alpha_{k}, \beta_{k}\right)$, then the system of intervals $\left(\alpha_{k}, \beta_{k}\right)$, with $k=1,2, \cdots, n$, is the sought system of intervals. If $E$ is not contained in $\bigcup_{k=1}^{n}\left(\alpha_{k}, \beta_{k}\right)$, let $j$ be the smallest integer such that $t_{j}$ is not in $\bigcup_{k=1}^{n}\left(\alpha_{k}, \beta_{k}\right)$ (clearly, $\left.j \geqq n\right)$. Then, by the continuity of $f$ and $g$ at $t_{j}$, there exists an interval $\left(\alpha_{n+1}, \beta_{n+1}\right)$, with $a \leqq \alpha_{n+1}<t_{j}<\beta_{n+1} \leqq b$, such that both

$$
\begin{gathered}
\left|f\left(\beta_{n+1}\right)-f\left(\alpha_{n+1}\right)\right|<\frac{\varepsilon}{2^{n+1}}, \\
g\left(\beta_{n+1}\right)-g\left(\alpha_{n+1}\right)<\frac{\varepsilon}{2^{n+1}}
\end{gathered}
$$

and, also, both $\alpha_{n+1}, \beta_{n+1}$ are not equal to any $t_{k}, k=1,2, \cdots$. The sequence of intervals $\left(\alpha_{k}, \beta_{k}\right), k=1,2, \cdots$, constructed in this way, satisfies the requirements of hypothesis $\left(3^{\prime}\right)$.

REMARK 2. If the function $f$ is absolutely continuous on $[a, b], g(x) \equiv x$, and if $E$ is a set of measure zero, then $\left(3^{\prime}\right)$ is satisfied (with $g(x) \equiv x$ ). More generally, if, for every $\varepsilon>0$, there exists a positive $\delta$ such that, for every system of open disjoint intervals $\left(c_{k}, d_{k}\right), k=1,2, \cdots$, with $c_{k}<d_{k}$ and $\Sigma g\left(d_{k}\right)-g\left(c_{k}\right)<\delta$, the inequality $\Sigma\left|f\left(d_{k}\right)-f\left(c_{k}\right)\right|<\varepsilon$ holds (absolute continuity of $f$ with respect to $g$ ); and the set $E$ is of $g$-measure zero, then hypothesis $\left(3^{\prime}\right)$ is satisfied.

\section{Vector valued functions}

The two theorems in this section can be described as "generalized mean value theorems of the differential calculus of vector valued functions", in the following sense: each theorem is concerned with a vector valued function $x(t)$, defined on a finite, closed, real number interval $[a, b]$; the hypotheses of each theorem state that the "small" difference quotients of the function $x(t)$ ("small" means that these difference quotients are taken over intervals interior to $[a, b]$ ) are "not too far away" from a certain set $C$; the conclusion of each theorem asserts that, in these circumstances, the "total", or "large" difference quotient of the function $x(t)$ ("large", means that this difference quotient is taken over the whole interval $[a, b])$ must actually, also, belong to the same set $C$.

THEOREM 3. If

(1) $X$ is a real linear locally convex topological space; 
(2) $x$ is a function which maps the interval $[a, b]$, with $a<b$, into $X$, and $x$ is weakly continuous at $a$ and $b$, from the right and from the left, respectively;

(3) $g$ is a real valued continuous function on $[a, b]$, with a finite derivative, $g^{\prime}(t)>0$ for $a<t<b$;

(4) $C$ is a closed, convex set, $C \subset X$, and the zero vector $0 \in C$;

(5) for every linear continuous functional $y$, on $X$ to the real numbers, there exists a set $E \subset(a, b)$, with the following properties (for brevity, the dependence of $E$ upon $y$ is not indicated explicitly):

(a) the real valued function $f(t)=y(x(t))$ has a, finite or infinite, derivative for $t \in(a, b)-E$,

( $\beta$ ) for every positive $\varepsilon$, there exists a sequence of open disjoint intervals $\left(\alpha_{k}, \beta_{k}\right), k=1,2, \cdots$, with $\alpha_{k}<\beta_{k}$ and $\left[\alpha_{k}, \beta_{k}\right] \subset[a, b]$, such that

$$
E \subset \bigcup_{k=1}^{\infty}\left(\alpha_{k}, \beta_{k}\right) \text { and, also, } \sum_{k=1}^{\infty}\left|f\left(\beta_{k}\right)-f\left(\alpha_{k}\right)\right|<\varepsilon,
$$

( $\gamma)$ for every $t \in(a, b)-E$, there exists a sequence of real numbers $\tau_{k}$, with $\tau_{k} \rightarrow 0$, and a corresponding sequence of vectors $z_{k} \in C$, such that

$$
y\left(\frac{x\left(t+\tau_{k}\right)-x(t)}{g\left(t+\tau_{k}\right)-g(t)}-z_{k}\right) \rightarrow 0
$$

as $k \rightarrow \infty$;

then

$$
\frac{x(b)-x(a)}{g(b)-g(a)} \in C
$$

Proof. Since $X$ is locally convex, $C$ is the intersection of all closed halfspaces which contain $C$. It will now be shown that the vector $\frac{x(b)-x(a)}{g(b)-g(a)}$ lies in every closed half-space which contains $C$; therefore, this vector must also belong to $C$.

Let $H$ be a closed half-space, $C \subset H$; then $H$ is known to be the set of all $z \in X$ such that

$$
y(z) \leqq M,
$$

where $y$ is a certain continuous linear functional on $X$, and $M$ is a real number. Since the vector $0 \in C$, it follows, from (7), that the number $0 \leqq M$. It follows, using hypothesis $5(\gamma)$, that, for every $t \in(a, b)-E$, \{recall that $f(t)=y(x(t))\}$ :

$$
\frac{f^{\prime}(t)}{g^{\prime}(t)} \leqq M
$$


and, hence, by Theorem 1, applied to the real valued function $f(t)=y(x,(t))$, on the interval $a \leqq t \leqq b$, it is also true that

$$
\frac{f(b)-f(a)}{g(b)-g(a)} \leqq M
$$

i.e., since $y$ is a linear functional,

$$
y\left(\frac{x(b)-x(a)}{g(b)-g(a)}\right) \leqq M ;
$$

i.e., by the definition of the closed half space $H$,

$$
\frac{x(b)-x(a)}{g(b)-g(a)} \in H \text {. }
$$

The proof is complete.

The counterpart of Theorem 2, for vector valued functions, is:

THEOREM 4. If hypotheses (1), (2), (3), of Theorem 3 are satisfied; and if, further,

(4') $C$ is a closed, convex set, $C \subset X$;

$\left(5^{\prime}\right)$ for every linear continuous functional $y$, on $X$ to the real numbers, there exists a set $E \subset(a, b)$ with the following properties (for brevity, the dependence of $E$ upon $y$ is not indicated explicitly):

$(\alpha)$ the real valued function $f(t)=y(x(t))$ has a, finite or infinite, derivative for $t \in(a, b)-E$,

( $\beta$ ) for every $\varepsilon>0$, there exists a sequence of open disjoint intervals $\left(\alpha_{k}, \beta_{k}\right), k=1,2,3, \cdots$, with $\alpha_{k}<\beta_{k}$, and $\left[\alpha_{k}, \beta_{k}\right] \subset[a, b]$, such that $E \subset \bigcup_{k=1}^{\infty}\left(\alpha_{k}, \beta_{k}\right)$, and, also, such that both $\sum_{k=1}^{\infty}\left|f\left(\beta_{k}\right)-f\left(\alpha_{k}\right)\right|<\varepsilon$ and $\sum_{k=1}^{\infty} g\left(\beta_{k}\right)-g\left(\alpha_{k}\right)<\varepsilon$,

( $\gamma)$ for every $t \in(a, b)-E$, there exists a sequence of real numbers $\tau_{k}$, with $\tau_{k} \rightarrow 0$, and a corresponding sequence of vectors $z_{k} \in C$, such that (6) holds; then

$$
\frac{x(b)-x(a)}{g(b)-g(a)} \in C .
$$

Theorem 4 can be proved in the same way as Theorem 3 , the only difference being that one uses Theorem 2, instead of Theorem 1, for the proof of (8).

(In comparing Theorems 4 and 3, it should be noticed that hypothesis (4') is weaker than hypothesis (4), while hypothesis $\left(5^{\prime}\right)$ is stronger than hypothesis (5).)

Remark 3. The condition $S^{\prime}(\beta)$ is satisfied, if, for example, $E$ is at most countable, and $f$ is continuous on $[a, b]$. This is a consequence of Remark 1. 
Remark 4. Condition $5^{\prime}(\beta)$ is also satisfied, with $g(t) \equiv t$, if $f$ is absolutely continuous on $[a, b]$, and $E$ is of measure zero. Condition $5^{\prime}(\beta)$ is also satisfied if $f$ is absolutely continuous with respect to $g$, and the set $E$ is of $g$-measure zero. This is all a consequence of Remark 2.

REMARK 5. If one and the same set $E$ can be chosen for all the functionals $y$; for example, if the vector valued function $x$ is weakly differentiable in $(a, b)-E$, and $5^{\prime}(\beta)$ and $5^{\prime}(\gamma)$ [or $5(\beta)$ and $5(\gamma)$ ] are satisfied for this particular ("universal", as far as the functional $y$ is concerned) set $E$, then, compare hypothesis (1), the function $g$ need not be differentiable for $t \in E$, and the conclusion of Theorem 4 [or Theorem 3] still holds.

\section{References}

A. K. Aziz and J. B. Diaz (1963), 'On a mean value theorem of the differential calculus of vector valued functions', Contributions to Differential Equations 1, 251-269.

V. I. Averbuch and O. G. Smoljanov (1967), 'Differential calculus in linear topological spaces', (Russian), Usp. Mat. Nauk, 22 specially pages 201-2.

J. B. Diaz and R. Výborný (1964), 'On mean value theorems for strongly continuous vector valued functions', Contributions to Differential Equations 3, 107-118.

J. Dieudonne (1960), Foundations of Modern Analysis (New York, 1960), specially pages 153-156.

R. M. McLeod (1964-65), 'Mean value theorems for vector valued functions', Proc. Edinburgh Math. Soc. 14, 197-209.

W. Mlak (1957), 'Note on the mean value theorem', Ann. Pol. Math. 3, 29-31.

R. M. J. Moss and G. T. Roberts (1968), 'A creeping lemma', Amer. Math. Monthly 75, 649-652.

T. Wazewski (1953), 'Une generalisation des théorèmes sur les accroissements finis', Ann. de la Societé Polonaise de Mathématiques 24, 132-147.

Rensselaer Polytechnic Institute

Troy, New York 12181, U.S.A.

University of Queensland

St. Lucia, 4067, Australia. 